\title{
Cross infection control measures and the treatment of patients at risk of Creutzfeldt Jakob Disease in UK general dental practice
}

\author{
J. Bagg, ' C. P. Sweeney, ${ }^{2}$ K. M. Roy, ${ }^{3}$ T. Sharp, ${ }^{4}$ and A. Smith, ${ }^{5}$
}

\begin{abstract}
Aims To determine the suitability of key infection control measures currently employed in UK dental practice for delivery of dental care to patients at risk of prion diseases.

Materials and methods Subjects: Five hundred dental surgeons currently registered with the General Dental Council of the UK. Data collection: Structured postal questionnaire. Analysis: Frequencies, cross-tabulations and chi-squared analysis. Results The valid response rate to the questionnaire was $69 \%$. $33 \%$ of practices had no policy on general disinfection and sterilisation procedures. Only 10 of the 327 responding practices (3\%) possessed a vacuum autoclave. $49 \%$ of dentists reported using the BDA medical history form but less than $25 \%$ asked the specific questions recommended by the BDA to identify patients at risk of iatrogenic or familial CJD. However, $63 \%$ of practitioners would refer such patients, if identified, to a secondary care facility. Of the 107 practitioners who were prepared to provide dental treatment, $75(70 \%)$ would do so using routine infection control procedures.

Conclusions Most of the dental practices surveyed were not actively seeking to identify patients at risk of prion diseases. In many cases, recommended procedures for providing safe dental care for such patients were not in place.
\end{abstract}

proteins are remarkably stable and resistant to most conventional sterilisation measures. ${ }^{5}$ Iatrogenic transmission of human prion diseases via neurosurgical instruments has been reported ${ }^{6}$ and there is some evidence that transmission via other surgical procedures may also occur. ${ }^{7}$

The potential risk of transmission of CJD in dentistry is unclear. Animal models have demonstrated the possibility of transmission of prions via the oral route, ${ }^{8-10}$ but current epidemiological evidence largely excludes a correlation between dental surgery and CJD. ${ }^{7,11,12}$ However, small clusters of CJD cases possibly connected by dental procedures have been reported. ${ }^{6,13}$ Whilst a recent paper has been unable to detect prion protein in dental pulp from patients with sporadic CJD, ${ }^{14}$ vCJD differs from the sporadic, iatrogenic and familial forms, in that the lymphoreticular tissues of vCJD cases are consistently infected with prion proteins. ${ }^{15}$ Further work is required to determine the infectivity of human pulpal and gingival tissues.

A Fact File, ${ }^{16}$ based on current recommendations from the Spongiform Encephalopathy Advisory Committee (SEAC), has been issued by the BDA. This recommends using the medical history form to identify patients who may be at risk of iatrogenic or familial forms of CJD. Questions enquire about previous

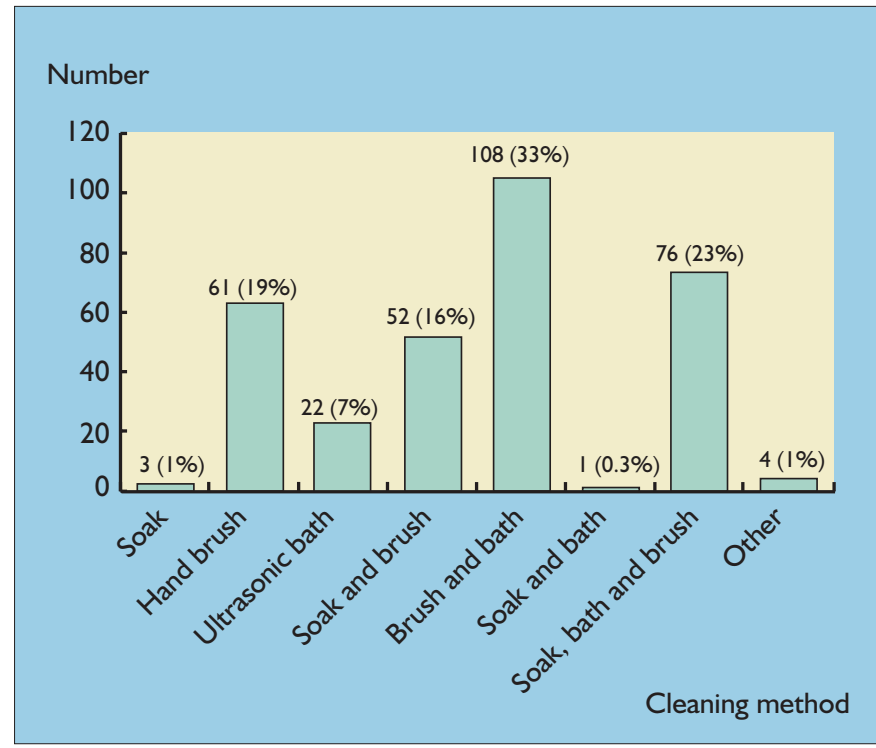

Fig. I The methods employed by practitioners for the cleaning of dental instruments prior to sterilisation 


\section{RESEARCH cross infection control}

brain surgery (in order to identify possible recipients of dura mater grafts); growth hormone use before the mid-1980s (after which artificially synthesised growth hormone came into use); and close family members who might have had CJD (to identify those at risk of familial CJD). ${ }^{16}$ These questions will not, however, identify those at risk of sporadic or vCJD. Patients who are identified as being at risk should either be treated with disposable instruments which are subsequently incinerated, or nondisposable instruments which should pass through a stringent decontamination process, separately from other instruments. This involves two washing cycles and a total of 18 minutes in a porous load (vacuum) autoclave. ${ }^{16}$ These recommended procedures represent significant changes to infection control practices for dental surgeons.

The present study had three aims. The first was to determine the proportion of dental practitioners who were using the recommended questions in the BDA Medical History Form. The second was to assess whether the decontamination and sterilisation procedures currently used in general dental practice would satisfy the criteria laid down by the SEAC. The final aim was to examine the willingness of dental surgeons to treat those at risk of CJD.

\section{Materials and method}

\section{Study participants}

Five hundred dental surgeons were selected at random from those registered in the United Kingdom with the General Dental Council (GDC). The names and addresses of these practitioners were then downloaded from the GDC web site.

\section{Questionnaire}

The questionnaire was divided into three sections. The first collected demographic data including sex, age band and sphere of dental practice (general dental practitioner, community dental practitioner or hospital/university dental service).

The second section related to decontamination and sterilisation procedures. The questions inquired if there was a written disinfection and sterilisation policy for the surgery or clinic; whether matrix bands, steel burs, endodontic instruments, mouthwash beakers, aspirator tips and scalpels were viewed as single use items; the methods used for cleaning instruments prior to sterilisation; the type and age of autoclave(s) used; and finally, the frequency of testing of autoclaves. No details were sought on the methods used for autoclave testing.

The third section sought information on whether practitioners used the BDA recommended questions in their Medical History Form to identify those at risk of CJD, and how frequently the medical histories of regular patients were updated. Finally, the practitioners were asked whether they would be prepared to treat patients at risk of CJD and, if so, what precautions they would take to prevent transmission.

\section{The survey}

The questionnaires were sent out in June 2000. A detailed covering letter, which explained the background to the study and stressed that the survey was completely anonymous, accompanied each questionnaire. A reply paid envelope was enclosed for return of the completed questionnaire. Reminder letters, containing a further copy of the questionnaire and another reply-paid envelope, were mailed in August 2000.

\section{Data analysis}

Data were analysed using SPSS (Statistical Package for the Social Sciences). A database was established in SPSS. Frequencies were used to examine the distribution of responses for all the variables and to describe sample demographics. The association between variables was examined by cross-tabulations and the

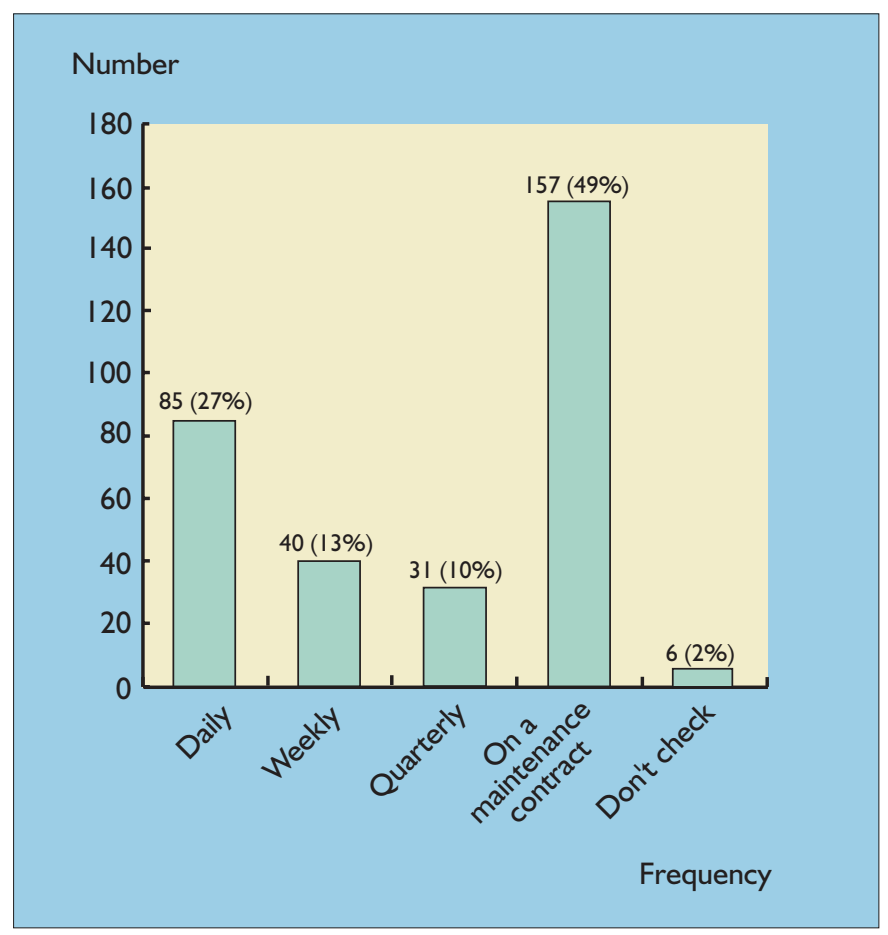

Fig. 2 The frequency of testing of autoclaves in the dental practices

statistical significance of such relationships examined by chisquared analysis.

\section{Results}

\section{Respondents}

Two of the 500 questionnaires were returned by the Post Office as undelivered. A total of 327 returned questionnaires were useable. A further 26 practitioners returned questionnaires indicating that they had retired from dentistry, and four questionnaires were returned blank. This represents an overall valid response rate of $327 / 472(69 \%)$.

Of those providing useable responses, $236(73 \%)$ were male. The age distribution was 55 (17\%) 21-30 years; 112 (34\%) 31-40 years; 102 (31\%) 41-50 years, 45 (14\%) 51-60 years and 13 (4\%) more than 60 years old. The group comprised $280(86 \%)$ general dental practitioners, 29 (9\%) community dental surgeons and $15(5 \%)$ hospital/university dentists.

Chi-squared analyses showed no effects of age or sex on the parameters reported below.

\section{Sterilisation and disinfection practices}

Of those responding, 217 (67\%) had a written policy detailing the disinfection and sterilisation procedures to be followed. Of those for whom no such policy was available, $98 \%$ were in general dental practice.

The majority of respondents re-processed matrix bands (88\%), steel burs (75\%) and endodontic instruments (88\%) after use. Disposable aspirator tips were used by $59 \%$ and the majority used disposable mouthwash beakers (96\%) and scalpels (97\%).

The frequency of use of the various methods for cleaning instruments prior to sterilisation is shown in Figure 1. Whilst a wide range of combinations were employed, hand brushing was an element of the cleaning reported by 297 (91\%) of those responding.

The age of the autoclaves in the practices varied from brand new to 28 years old (mean $6.8 \pm 5.1$ years). Ten of the 327 responding practices $(3 \%)$ possessed a vacuum autoclave. Figure 2 illustrates 


\section{RESEARCH cross infection control}

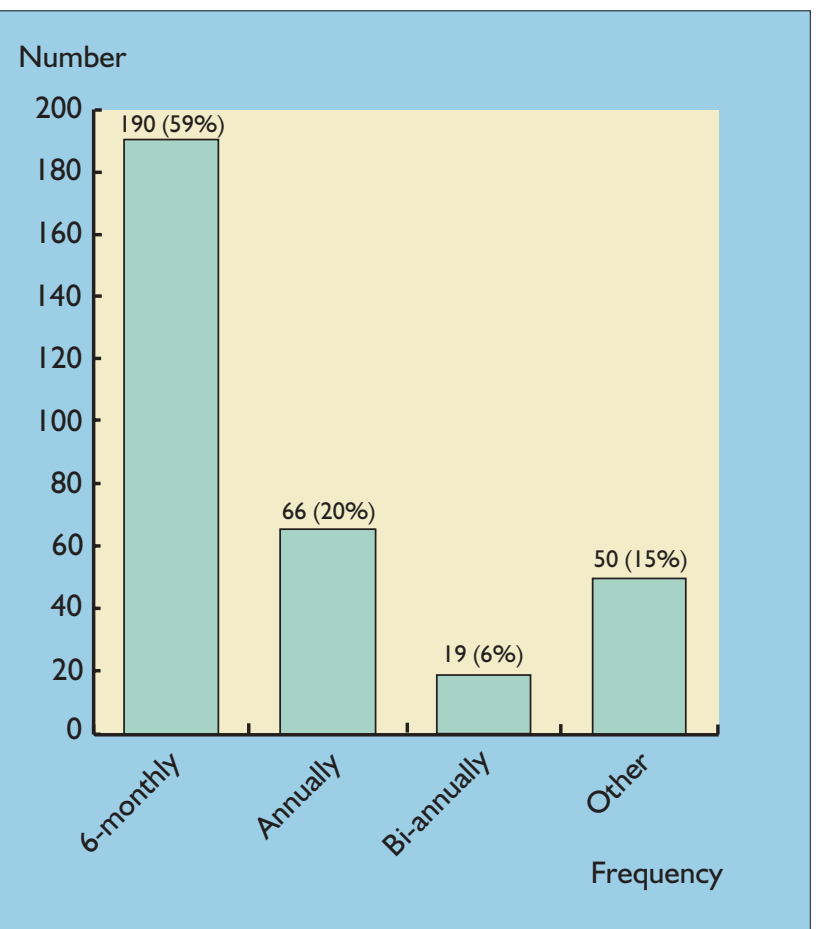

Fig. 3 The frequency of updating of dental patients' medical histories

the frequency of autoclave testing undertaken. Only a small proportion of practitioners $(2 \%)$ undertook no checks whatsoever and $49 \%$ had a commercial maintenance contract.

\section{Medical history form}

The Medical History Form, recommended by the BDA in 1998, was reportedly used by $49 \%$ of the respondents. However, only a minority of dentists used the specific questions related to identifying those at risk of iatrogenic and familial CJD. Thus, $24 \%$ enquired about previous brain surgery, $20 \%$ asked about treatment with growth hormone and $18 \%$ asked patients whether they had a close relative with CJD. More than three-quarters of respondents (79\%) had a policy of updating medical histories at least annually (Fig. 3).

As far as management of a patient identified to be at risk of CJD was concerned, a high proportion (63\%) would refer the case to a secondary care facility (Fig. 4). Of the 107 (33\%) practitioners who were prepared to provide dental treatment, 75 (70\%) would do so using routine infection control procedures, while $32(30 \%)$ would incinerate the dental equipment used (Figure 4). Of the latter, 11 practitioners would then refer the patient on for continuation of care.

\section{Discussion}

The discovery of variant CJD, ${ }^{2}$ together with widespread scientific and media interest in this disease, have heightened awareness of the risk of transmission of prions in the healthcare setting. The number of cases of VCJD in the UK is still low (95 definite and probable cases to 2 March 2001), and a preliminary screening study of vCJD reactivity in around 3000 archived tonsils and appendix specimens revealed no positive results. ${ }^{17}$ However, there is still concern among many experts that an epidemic, linked to consumption of meat from BSE-infected herds, may develop. ${ }^{18}$ It is, therefore, important that the dental profession gives serious thought to provision of safe dental care, in the light of the resistance of prions to traditional sterilisation procedures. ${ }^{5}$ The findings of the present study suggest that general dental practice is not currently equipped to address some of these issues.

It is of concern that $33 \%$ of respondents had no practice policy on disinfection and sterilisation procedures. Irrespective of the CJD problem, dental practices must show a commitment to decontamination and sterilisation; a written policy, understood by all the dental team is viewed as a pre-requisite.

Most of the practices reprocessed steel burs, matrix bands and endodontic instruments and the majority cited cleaning followed by autoclaving as their routine decontamination procedure. This is a logical process, but it must be remembered that these items are difficult to clean because of their complex surface contours. In relation to sterilisation in general, but prion decontamination in particular, initial cleaning is an essential stage of instrument reprocessing ${ }^{19}$ and great care must be taken to perform the cleaning thoroughly. Many combinations of methods were reported for the cleaning of instruments, but most $(91 \%)$ of these involved an element of hand scrubbing. In a recent study of matrix band cleaning (submitted for publication) ultrasonic baths were significantly more effective than hand scrubbing for removal of blood contamination. It is also important that dental nurses are aware of the need for strong gloves and to take care to avoid sharps injuries during hand cleaning of instruments.

Ten practices had access to a vacuum autoclave, whilst all the remaining practices used downward displacement autoclaves. Although acceptable for the routine sterilisation of unwrapped instruments, downward displacement autoclaves are not deemed appropriate for inactivation of prions. ${ }^{16}$ Thus, for the vast majority of dental practices in the UK, any instruments used in the treatment of patients at risk of CJD would have to be incinerated. Practitioners replacing old autoclaves should consider purchase of a vacuum model.

The questionnaire did not identify whether or not respondents were BDA members. However, the survey indicated that a high proportion of UK dentists were not making use of the new BDA recommended medical history form (released 1998). The form contains three questions designed to identify patients at risk of iatrogenic or familial CJD. In reality, it appears that few dentists ask these specific questions, even if they are using a $\mathrm{BDA}$ recommended medical history form, though many believed that the more general questions they used would identify those who had undergone brain surgery or received growth hormone injections. Most of the practitioners updated patients' medical histories at least annually, but since few are actively seeking out patients in the 'at risk' group, it is unlikely that large numbers will be identified in dental practice. Nevertheless, more than $60 \%$ of dentists indicated that if such patients were identified, they would refer them to a secondary care facility, as currently recommended in the BDA Fact File. ${ }^{16}$ Of those prepared to provide treatment for such patients in the practice, $70 \%$ would use routine infection control procedures. This contravenes the current official guidelines, namely that instruments used for treating such patients should either be incinerated or undergo stringent decontamination, the latter proving impossible in most dental practices because of the lack of vacuum autoclaves. Many respondents commented on the questionnaire that they would need to seek advice. It is important that dentists are aware of how to access appropriate expertise and full details are given in the Department of Health publication 'Creutzfeldt-Jakob Disease: Guidance for Healthcare Workers'.

The prion problem is a significant potential complication for infection control policy making in dentistry. There are still many unknowns in the equation, particularly the level of prion infectivity (if any) in human oral and dental tissues. The more widespread tissue distribution of $\mathrm{vCJD}$ compared with the classic forms ${ }^{15}$ is a concern and work is urgently needed to examine oral tissues for the agent of vCJD. There are no questions which can be asked in the medical history that will identify those infected with vCJD and present knowledge does not indicate whether the number of such patients is small, or runs into thousands. Until further information 


\section{RESEARCH

Fig. 4 Responses to the question regarding the procedures for providing dental care for patients identified as 'at risk' of prion diseases

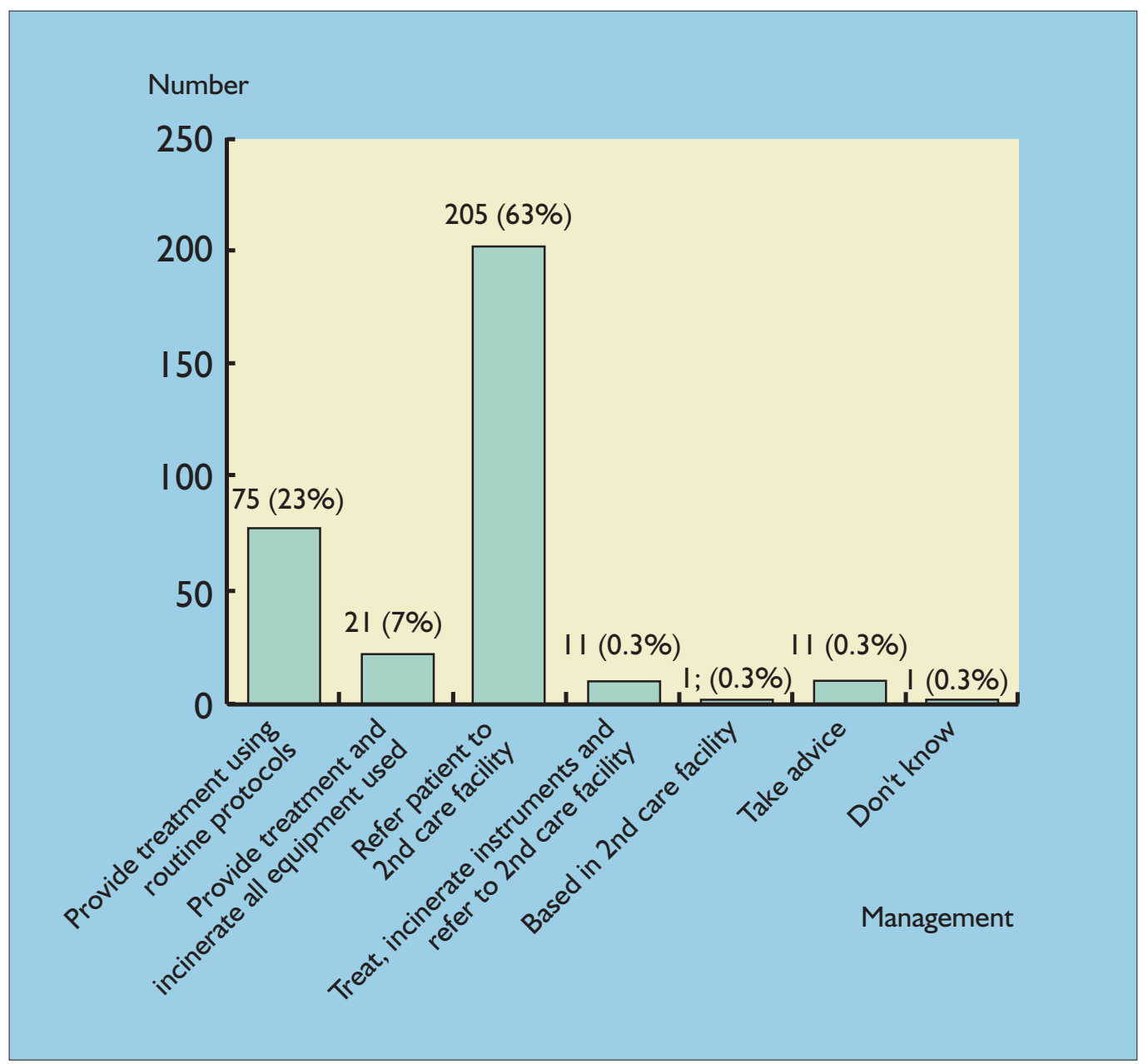

becomes available, dentists, along with all other healthcare workers, must ensure that they are applying the principles of universal infection control rigorously and, in particular, that instrument cleaning prior to sterilisation is undertaken thoroughly.

The authors would like to thank all those dental surgeons who kindly completed and returned the questionnaire.

1. Johnson R T, Gibbs C J. Medical progress: Creutzfeldt-Jakob Disease and related transmissible spongiform encephalopathies. N Engl J Med 1998; 339: 1994-2004

2. Will R G, Ironside J W, Zeidler M et al. A new variant of Creutzfeldt-Jakob Disease in the UK. Lancet 1996; 347: 921-925.

3. Hill A F, Desbruslais M, Joiner S, Sidle K C L, Gowland I, Collinge J. The same prion strain causes vCJD and BSE. Nature 1997; 389: 448-450.

4. Bruce M E, Will R G, Ironside J W et al. Transmissions to mice indicate that 'new variant' CJD is caused by the BSE agent. Nature 1997; 389: 498-501.

5. Taylor D M. Inactivation of prions by physical and chemical means. J Hosp Infect 1999; 43 (suppl): S69-S76.

6. Will R G, Matthews W B. Evidence for case-to-case transmission of Creutzfeldt-Jakob disease. I Neurol Neurosurg Psych 1982; 45: 235-238.

7. Collins S, Law M G, Fletcher A, Boyd A, Kaldor J, Masters C L. Surgical treatment and risk of sporadic Creutzfeldt-Jakob disease: a case-control study. Lancet 1999; 353: 693-697.

8. Adams D H, Edgar W M. Transmission of agent of Creutzfeldt-Jakob disease. Br Med J 1978; 1: 987.
9. Carp R I. Transmission of scrapie by oral route: effect of gingival scarification. Lancet 1982; 16: 170-171.

10. Ingrosso L, Pisani F, Pocchiari M. Transmission of the $263 \mathrm{~K}$ scrapie strain by the dental route. J Gen Virol 1999; 80: 3043-3047.

11. Davanipour Z, Alter M, Sobel E, Asher D M, Gajdusek D C. CreutzfeldtJakob disease: possible medical risk factors. Neurol 1985; 35: 1483-1486

12. Van Duijn C M, Delasnerie-Laupretre N, Masullo C, Zerr I, de Silva R, Wientjens D P W M et al. Case-control study of risk factors of CreutzfeldtJakob disease in Europe during 1993-95. Lancet 1998; 351: 1081-1085.

13. Arakawa K, Nagara H, Itoyama Y, Doh-ura K, Tomokane N, Tateishi J, Goto I. Clustering of three cases of Creutzfeldt-Jakob disease near Fukuoka City, Japan. Acta Neurol Scand 1991; 84: 445-447.

14. Blanquet-Gossard F, Sazdovitch V, Jean A, Deslys J-P, Dormont D, Hauw $\mathrm{J}-\mathrm{J}$ et al. Prion protein is not detectable in dental pulp from patients with Creutzfeldt-Jakob disease. J Dent Res 2000; 79: 700.

15. Hill A F, Butterworth R J, Joiner S et al. Investigation of variant CreutzfeldtJakob disease and other human prion diseases with tonsil biopsies. Lancet 1999; 353: 83-89.

16. British Dental Association. Fact File: Creutzfeldt-Jakob Disease. August 2000 .

17. Dobson R. First results of vCJD survey show no signs of prion. Br Med J 2000; 320: 1226

18. Ashraf H. Early results of study into vCJD epidemic inconclusive. Lancet 2000; 355: 1619-1626.

19. Laurenson I F, Whyte A S, Fox C, Babb J R. Contaminated surgical instruments and variant Creutzfeldt-Jakob disease. Lancet 1999; 354: 1823-1826. 